\title{
STUDY ON DETECTION TECHNOLOGY OF PESTICIDE RESIDUES IN VEGETABLES BASED ON NIR
}

\author{
Jingzhu Wu ${ }^{1, *}$, Cuiling Liu ${ }^{1}$, Yuanyuan $\mathrm{Chen}^{1}{ }^{1}$, Yan $\mathrm{Chen}^{1}$, Yun $\mathrm{Xu}^{2}$ \\ ${ }^{1}$ School of Information Engineering, Beijing Technology \& Business University, Beijing, \\ P. R. China 100037 \\ ${ }^{2}$ College of Information and Electrical Engineering, China Agricultural University, Beijing, \\ P. R. China 100083 \\ * Corresponding author, Address: School of Information Engineering, Beijing Technology \& \\ Business University, Beijing, 100037, P. R. China, Tel: +86-10-68985506, Email: \\ pubwu@163.com
}

Abstract: A quantitative technology based on near infrared spectroscopy (NIR) is proposed to detect pesticide (chlorpyrifos) residues in vegetables in the paper. There are three groups of samples (totally 100 unit, concentration from $0.1 \mathrm{mg} / \mathrm{kg}$ to $4 \mathrm{mg} / \mathrm{kg}$ ) used in the experiment. The first group sample is confected to simulate vegetable juice to validate feasibility of the NIR quantitative method. As shown in this work, the first NIR model has good indexes (R2 is 0.9807 , RMSEP is 0.149). Next the second group sample is confected by adding material standards of $1 \mathrm{mg} / \mathrm{ml}$ of chlorpyrifos to spinage juice. The second NIR model also has good performance (R2 is 0.9858 , RMSEP is 0.207 ). The third group sample is confected as the second group to validate repeatability of the detection method based on NIR. The third model is also excellent ( $\mathrm{R} 2$ is 0.9986, RMSEP is 0.0485). The exploratory experiment results indicate the detection technology of pesticide residue in vegetables based on NIR is feasible in the given conditions. But supposed to the natural vegetable samples that include pesticide residue, the method should to be validated more.

Keywords: $\quad$ NIR, Pesticide Residue, Detection, Spinage

Please use the following format when citing this chapter:

Wu, J., Liu, C., Chen, Y., Chen, Y. and Xu, Y., 2009, in IFIP International Federation for Information Processing, Volume 295, Computer and Computing Technologies in Agriculture II, Volume 3, eds. D. Li, Z. Chunjiang, (Boston: Springer), pp. 2217-2222. 


\section{INTRODUCTION}

The invention and use of pesticides has greatly enhanced the crop yield. However, with the widely and unreasonable use of pesticide the negative impact is increasingly exposed, especially poisoning incidents occur from time to time caused by pesticide residues in vegetables. The detection technology of pesticide residues gets great attention. At present there are some traditional chemical method to detect pesticide residues, such as GC, PLC and CG-MS (Wang, et al, 2006). But these methods are timeconsuming and complex. Otherwise chemical methods need consume chemical and destroy the estimate sample. So it's unfit for the national supervise department to execute the inspect task locally.

Near infrared spectrometry is fast developed after ninety in twentieth century in China. The NIR-based (Near Infrared Spectroscopy analysis) technology is recommended for agricultural products detection because it is a fast, lossless and sustainable technology. At present, Pierre Billeen had been using near-infrared detection of pesticides used in seed dressing, and achieved good results (Pierre, et al, 2000). Wen-Xiu Li, such as the use of high pesticide residues in vegetables dichlorvos trichlorfon and the infrared absorption of solvent juice, vegetables directly on the pesticide residue was detected (LI, et al, 2004). Xiang-Yang Zhou, analyzed a variety of vegetable samples near-infrared spectra of the differences in the use of phosphoruscontaining group characteristic absorption frequency of the area to identify, and GC-MS method than the right, made with the differential effects (ZHOU, et al, 2004).

Pesticide residues detection belongs to trace detection. This paper does exploratory studies about quantitative detection technology of pesticide (chlorpyrifos) residues in vegetables based on near infrared Spectroscopy.

\section{THEROY}

Near-infrared is the electromagnetic wave between the middle-infrared and visible. Its wavelength range is between $800 \mathrm{~nm}$ to $2500 \mathrm{~nm}$.

Near infrared spectroscopy is the absorption spectrum which is mainly obtained by organic molecules hydrogen group $(\mathrm{C}-\mathrm{H}, \mathrm{N}-\mathrm{H}, \mathrm{O}-\mathrm{H}$, etc.) stretching at all levels and frequency of these groups of stretching and bending of the frequency of vibration. The spectrum of areas include almost all of the hydrogen-containing organic compounds in the group, contains elements of the structure, composition status, and other information. The amount of information is extremely rich, so as to near-infrared spectral analysis of quantitative samples of the physical nature and chemical composition may be provided. 
There are many kinds of pesticide are widely used in China, especially organophosphorus pesticides. Chlorpyrifos is one kind of organophosphorus and it is always detected in leaf vegetable higher than national standard. The Molecular formula of chlorpyrifos is $\mathrm{C}_{9} \mathrm{H}_{11} \mathrm{Cl}_{3} \mathrm{NO}_{3} \mathrm{PS}$ including hydrogen group, which provide the theoretical basis for Near Infrared Spectroscopy analysis.

\section{EXPERIMENT}

\subsection{Sample preparation}

There are three group of samples designed in the experiment listed in table 1. The background solution of first group is simulation of pesticide residues in samples of vegetables. The components are includes sugar, Vitamin $\mathrm{C}$ and drinking water mixed solution. The samples with different pesticide residues can be obtained by adding material standards of $1 \mathrm{mg} / \mathrm{ml}$ of chlorpyrifos according to design requirements.

To approach natural samples the background solution of the second and third group is spinage juice. The spinage purchased from the supermarket is Organic vegetables without pesticide residues. The spinage juice is prepared in the Chinese Academy of Agricultural Sciences. The samples with different pesticide residues can be obtained like the first group.

Table 1. Sample preparation

\begin{tabular}{ccccc}
\hline Group Label Preparation time & Background Solution & Range of Concentration $(\mathrm{mg} / \mathrm{kg})$ & Number of Sample \\
\hline 1 & $2007-12-11$ & simulation vegetable juice & $0.1-3$ & 30 \\
2 & $2008-03-07$ & spinage juice & $0.1-4$ & 35 \\
3 & $2008-05-07$ & spinage juice & $0.1-4$ & 35 \\
\hline
\end{tabular}

\subsection{Spectral data}

The spectrums of the first group are collected by fiber Optic transmission from 12500 to $4000 \mathrm{~cm}^{-1}$ in $8 \mathrm{~cm}^{-1}$ on MPA spectrometer with the liquid fiber probe of $2 \mathrm{~m}$, the wavelength path of $1 \mathrm{~mm}$. The spectrums of the second and third group are collected on MATRIX-F spectrometer. The other parameters are same. Spectrum acquisition and analysis software used OPUS5.0. 


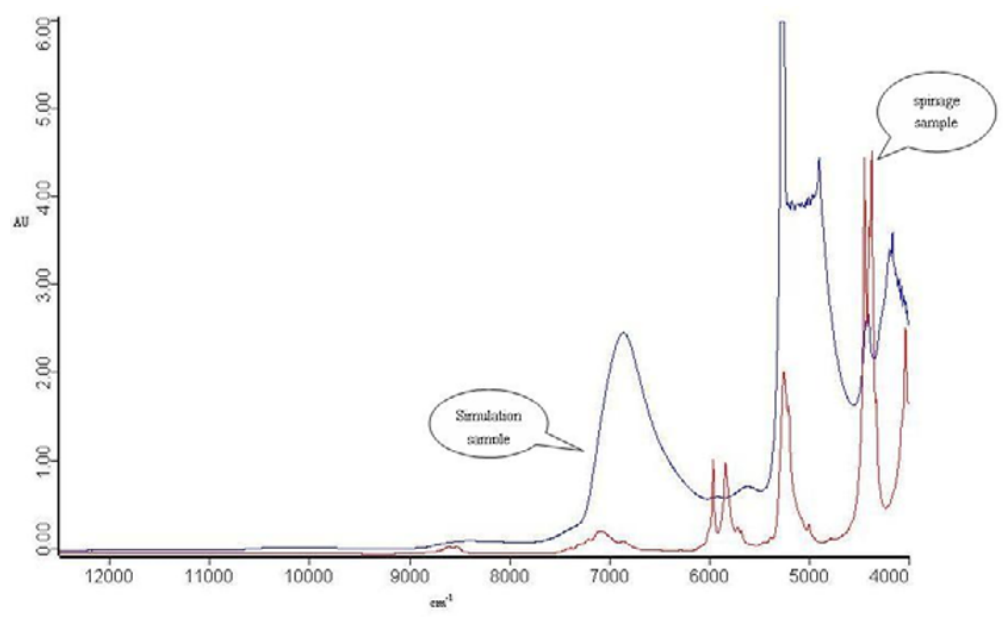

Fig. 1: 2 unit spectrums

\section{RESULTS AND DISCUSSION}

According to the concentration difference, the sample set is divided to train set and test set. The number of the train set and test set is approximately 3 . The result lists in the table 2 .

From the table 2 the NIR model built by simulation samples is good. The second NIR model built on the natural spinage juice samples also has good performance. The third model is built based on the third group samples to validate the last model's reliability. The result is also satisfied.

Table 2. NIR index of 3 groups of samples

\begin{tabular}{cccccc}
\hline Group Label & Train set & Test set & $\mathrm{R}^{2}$ & RMSECV & RMSEP \\
\hline 1 & 23 & 7 & 0.9807 & 0.123 & 0.149 \\
2 & 27 & 8 & 0.9858 & 0.154 & 0.207 \\
3 & 27 & 8 & 0.9986 & 0.0437 & 0.0485 \\
\hline
\end{tabular}

From the table 2 the NIR model built by simulation samples is good. The second NIR model built on the natural spinage juice samples also has good performance. The third model is built based on the third group samples to validate the last model's reliability. The result is also satisfied. The prediction of test sample of the second and third group list in Table 3 and Table 4.

Pesticide residues detection belongs to trace detection. It is very difficult to detect theoretically. But the result of the experiment in this paper is satisfied unexpectedly. Maybe the reason is that samples are made uniformity solution, which is conducive to collecting spectrum with strong signal, and all samples are ideal samples confected by manual in give some 
condition. That reduced sample complexity. The Study on quantitative detection technology of pesticide (chlorpyrifos) residue in vegetables based on near infrared Spectroscopy should be continued deeply.

Table 3. Prediction of test sample of Group 2

\begin{tabular}{cccc}
\hline Filename & True & Prediction & Difference \\
\hline 04.0 & 0.35 & 0.3307 & 0.0193 \\
08.0 & 0.55 & 0.7266 & -0.177 \\
12.0 & 0.8 & 0.9077 & -0.108 \\
16.0 & 1.1 & 0.8374 & 0.263 \\
20.0 & 1.5 & 1.644 & -0.144 \\
24.0 & 1.9 & 1.64 & 0.26 \\
28.0 & 2.5 & 2.873 & -0.373 \\
32.0 & 3.2 & 3.246 & -0.0456 \\
\hline Table 4. Prediction of test sample of Group 3 & & \\
\hline Filename & True & Prediction & Difference \\
\hline 04.0 & 0.35 & 0.3099 & 0.0401 \\
08.0 & 0.55 & 0.4504 & 0.0996 \\
12.0 & 0.8 & 0.7574 & 0.0426 \\
16.0 & 1.1 & 1.149 & -0.0486 \\
20.0 & 1.5 & 1.506 & -0.00582 \\
24.0 & 1.9 & 1.933 & -0.033 \\
28.0 & 2.6 & 2.643 & -0.043 \\
32.0 & 3.4 & 3.39 & 0.00996 \\
\hline
\end{tabular}

\section{CONCLUSION}

This paper discusses the quantitative detection technology of pesticide (chlorpyrifos) residue in vegetables based on near infrared Spectroscopy. The experimental results of three groups of samples indicate the detection technology of pesticide residue in vegetables based on NIR is feasible in the given conditions in this paper. But supposed to the natural vegetable samples that include pesticide residue, the method should to be validated more.

\section{ACKNOWLEDGEMENTS}

Funding for this research was provided by Beijing Natural Science Foundation, P. R. China (project No. 4073031). The authors would like to thank Doctor Yuan Yuwei from the Chinese Academy of Agricultural Sciences to help us confect the spinage samples. Deeply Tanks for BRUKER OPTICS corp. to supply the NI Spectrograph to help us collect samples' spectrum. 


\section{REFERENCES}

LI Wen-iu, XU Kexin. Investigation on the Detection of Pesticide Residue in Vegetable Based on Infrared Spectroscopy. Spectroscopy and Spectral Analysis, 2004, 24(10):12021204

Pierre Billeen, etal. Testing seeds protected with an insecticide by NIR. Proceeding of the 9th International Conference, 2000, 625-628.

Wang chaojing, Cai qi. Tendency of Detection of Residual Pesticides in Farm Produce. Modern Scientific Instruments, 2006, 01:106-108

ZHOU Xiang-yang, LIN Chung-zhon. Rapid Indentification of Organophosphorus Pesticide Residues in Leaf-vegetable by FT-NIR. Food Science, 2004, 25(05):151-154 